\title{
Stability Analysis of the Oscillatory Electrodissolution of Copper with Impedance Spectroscopy
}

\author{
István Zalán Kiss and Vilmos Gáspár* \\ Institute of Physical Chemistry, Kossuth Lajos University, H-4010 Debrecen, P.O. Box 7, Hungary
}

\author{
Lajos Nyikos* \\ Physical Chemistry Laboratory, Central Research Institute for Physics, H-1525 Budapest, \\ P.O. Box 49, Hungary
}

Received: June 16, 1997; In Final Form: October 15, 1997

\begin{abstract}
Impedance spectroscopy is applied to quantitatively characterize the bifurcations leading to current oscillations during anodic dissolution of a copper rotating-disk electrode in sodium acetate-glacial acetic acid and $o$-phosphoric acid electrolytes under potentiostatic control. The line of Hopf bifurcations in a diagram spanned by the uncompensated series resistance and the true electrode potential has been constructed by measuring the linear frequency response of the electrochemical systems. On the basis of impedance data, we have also determined the critical frequency by which oscillations emerge at the bifurcation points. Qualitative differences in the origin of current oscillations in the studied systems are explained by the results of experimental linear stability analysis with impedance spectroscopy.
\end{abstract}

\section{Introduction}

Since the first observation of current oscillations during electrodissolution of an iron wire in nitric acid by Fechner ${ }^{1}$ in 1828, the experimental evidence of electrochemical instabilities resulting in nonlinear behavior such as oscillations, multistability, and chaos have been constantly accumulating. A comprehensive review on nonlinear phenomena in electrochemical systems has been published recently by Hudson and Tsotsis. ${ }^{2}$ With the emergence of a new paradigm in chaos theory, namely that chaos can be controlled by applying small perturbations to a system constraint, ${ }^{3}$ the experimental and theoretical interest in electrochemical systems has been renewed. As electrochemical instabilities can be monitored by simple current or voltage measurements and parametric changes can be easily achieved by current or voltage control, electrochemical systems seem to be an ideal playground for testing different control strategies. The idea has been put into practice first by Parmananda et al. ${ }^{4-6}$ They reported on controlling the chaotic current oscillations during the anodic dissolution of a copper rotating-disk electrode (RDE) in sodium acetate-glacial acetic acid (SAGA) buffer. In a recent publication, ${ }^{7}$ we have reported on controlling the chaotic current oscillations during the electrodissolution of a copper RDE in o-phosphoric acid (OP) electrolyte. ${ }^{8-10}$

Although both systems show the same type of complex phenomena, we have observed that under identical experimental conditions (electrodes, electrochemical cell, rotation rate, temperature, etc.) periodic or chaotic current oscillations spontaneously appear in the $\mathrm{Cu}-\mathrm{SAGA}$ system; however, even simple oscillations would not occur in the $\mathrm{Cu}-\mathrm{OP}$ system without connecting a sufficiently large external resistor in series with the copper electrode. It is important to point out that this is not a general conclusion about these systems but rather holds for the experimental conditions used in our laboratory. For

* To whom correspondence should be addressed. example, Schell et al. ${ }^{8,9}$ did not apply an external resistor; thus, current oscillations in their cell were observed at very high rotation rates only.

In this paper, we shed light on the observed difference in the origin of current oscillations by the experimental linear stability analysis of the two systems with the help of impedance spectroscopy. In an electrochemical impedance measurement ${ }^{11}$ the potential applied between the working and reference electrodes is subject to small-amplitude (maximum of ca. 5 $\mathrm{mV}$ ) sinusoidal perturbations superimposed on some constant circuit potential $V$. Thus, the true electrode potential $E=V-$ $I R_{\mathrm{S}}$ and the current $I$ flowing through the electrode are also modulated. Here, $R_{\mathrm{S}}$ is the internal or external series resistance between the electrodes. The interfacial impedance $Z=\Delta E / \Delta I$ is measured over a large range of frequencies and presented in the complex impedance plane by plotting $\operatorname{Im}(Z)$ against $\operatorname{Re}(Z)$. Therefore, an impedance spectrum comprises all experimentally accessible information about the linear frequency response of an electrode in one plot.

The Nyquist theorem, ${ }^{12}$ which establishes the relation between the frequency response and stability of an electronic device, has been known for long in electric engineering. Reviews on application of impedance spectroscopy for linear stability analysis of electrochemical systems have been published recently by Koper. ${ }^{13-15}$ As he pointed out, one of the main advantages of impedance spectroscopy is that it allows one to determine the necessary conditions for the appearance of bifurcations leading to instabilities without the need to generate a nonequilibrium phase diagram of the system by systematically varying some control parameters over large ranges. In addition, linear stability analysis by impedance spectroscopy does not require a detailed kinetic model for the electrochemical processes. For example, according to the Nyquist stability criterion ${ }^{12}$ an electrochemical system under potentiostatic control will undergo a Hopf bifurcation, ${ }^{16}$ resulting in current oscillations if the total 
impedance $Z_{\mathrm{tot}}=Z+R_{\mathrm{s}}$ is found to be zero at a finite, nonzero $\omega$ frequency. Physically, it is easy to understand why zero impedance should correspond to a natural periodic response of the system. When the electrochemical cell is driven with an arbitrarily small input signal of this particular frequency, there is a resonance. The input signal does not experience any resistance or phase delay, and $\operatorname{Re}\left(Z_{\text {tot }}\right)=\operatorname{Im}\left(Z_{\text {tot }}\right)=0$.

In this paper, we report on the application of impedance spectroscopy to characterize the Hopf bifurcations leading to current oscillations during the anodic dissolution of a copper RDE in SAGA buffer and OP electrolytes under potentiostatic control. First, procedures and apparatus are described in the experimental section. Then we present the results of impedance measurements and determine the line of Hopf bifurcations in a diagram spanned by the uncompensated series resistance and the true electrode potential. We conclude by analyzing the qualitative difference in the origin of instabilities in the studied electrochemical systems.

\section{Experiments}

Experiments were performed by using a standard threeelectrode electrochemical cell equipped with a copper RDE, a saturated calomel electrode (Radelkis OH-0933P) as reference, and a Pt-sheet counter electrode (Radelkis $\mathrm{OH}-9437$, area 5 $\mathrm{cm}^{2}$ ). All potentials are given with respect to the SCE. The cell was thermostated at $24 \pm 0.1{ }^{\circ} \mathrm{C}$. A small cut of a copper rod $(5 \mathrm{~mm}$ diameter) was fixed in the Teflon holder of a Tacussel RDE apparatus. The surface of the copper electrode (99.99\% purity) has been freshly polished by a series of wet sanding. The rotation rate was kept constant, $1000 \mathrm{rpm}$, in all reported experiments. The applied SAGA buffers were freshly prepared by mixing $50.0 \mathrm{~mL}$ of $2.0 \mathrm{M}$ sodium acetate (Reanal) solution and $90.0 \mathrm{~mL}$ of glacial acetic acid (Erdökémia, 96\%). $o$-Phosphoric acid (Merck or Reanal, 85\%) was used as received.

The cell was connected to a Solartron 1286 potentiostat. Impedance values were measured by a Solartron 1250 frequencyresponse analyzer coupled to the potentiostat. The output current signal of the potentiostat was fed into a high-speed voltmeter (Keithley 194A) and a digitizing oscilloscope (HewlettPackard 54561A). The digitized data were stored on a PC for later retrieval and plotted by a Hewlett-Packard 7090A plotter. All instruments were controlled by the PC via the GPIB-bus.

Impedance measurements in the frequency range $10^{-2}-10^{4}$ $\mathrm{Hz}$ were performed as follows. First, the high-frequency range of an impedance spectrum was measured at $0.0 \mathrm{mV}$ electrode potential where the studied systems are unconditionally stable. The series resistance $R_{\mathrm{s}}$ of a given system was determined by linearly extrapolating the high-frequency part of the impedance plot to infinite frequency where $\operatorname{Im}\left(Z_{\text {tot }}\right)=0$. During impedance measurements this resistance has been always compensated by using the positive-feedback (built-in) circuit of the potentiostat. The $I R$ compensation has two advantages: (i) the system becomes stable, which is a necessary condition for impedance measurement anyway, and (ii) the potential at the electrode/ solution interface is constant and independent of the current flowing through it. All potential values reported in this paper are compensated, true electrode potentials.

For the $\mathrm{Cu}-\mathrm{SAGA}$ system a special treatment of the copper electrode had to be developed in order to obtain reproducible results. The need for this procedure may originate from the slow relaxation of the system to its equilibrium, which could be also observed during simple voltammetric measurements. Figure 1 shows a typical polarization curve of the $\mathrm{Cu}-\mathrm{SAGA}$ system recorded at low scan rate. The first part of the curve

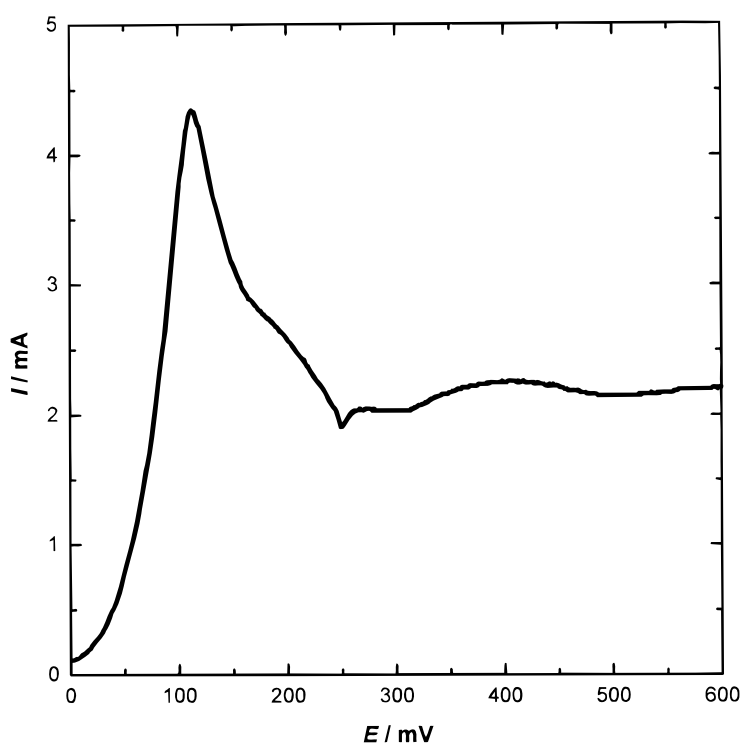

Figure 1. Polarization curve of a copper RDE in a mixture of 50.0 $\mathrm{mL}$ of $2.0 \mathrm{M}$ sodium acetate and $90.0 \mathrm{~mL}$ of glacial acetic acid. Compensated resistance $R_{\text {comp }}$ is equal to the series resistance of the cell $\left(R_{\mathrm{s}}=70.0 \Omega\right)$, rotation rate is $1000 \mathrm{rpm}$, and scan rate is 0.10 $\mathrm{mV} / \mathrm{s}$.

(from 0 to $300 \mathrm{mV}$ ) was always reproducible, but the position and height of the shallow maximum (at ca. $400 \mathrm{mV}$ ) changed from one experiment to the next. Apparently, even at this low scan rate the system has not reached steady state. The treatment of the copper electrode consists of the following steps. First, the series resistance of the cell is determined by impedance measurements at $0 \mathrm{mV}$ as described earlier. After compensating for this resistance at $0 \mathrm{mV}$, the true potential is set to $200 \mathrm{mV}$ and kept there for about $20 \mathrm{~min}$. During this period a peculiar phenomenon takes place. First, a blue ring appears at the edge of the electrode, which then starts to travel toward the shining center. Since the inner diameter of the blue ring decreases faster than the outer diameter, the ring is thickening as it travels inward. Meanwhile, the surface of the electrode behind the ring turns light-brownish. As these spatiotemporal processes take place-most probably due to the inhomogeneous potential distribution on the electrode surface-the total current monotonically increases. However, when the inner part of the blue ring reaches the center (and the ring turns into a disk), the current starts to decrease. As the current further decreases, the blue disk gradually changes-starting from the center-into darkbrownish. We have found that once the current started to decrease, the true electrode potential can be safely set to any desired higher (more anodic) value, and fairly reproducible behavior is observed. If the anodic potential is prematurely set to a higher value or no compensation is applied, either no oscillations are observed in the uncompensated system or the current oscillations are highly irregular and irreproducible.

\section{Results and Discussion}

Figure 2 shows the impedance spectra of the $\mathrm{Cu}-\mathrm{SAGA}$ system measured at different true potentials. Note that $-\operatorname{Im}(Z)$ is used for the convenience of plotting only. Since impedance measurements were performed under potentiostatic control with full $I R$ compensation, $R_{\text {comp }}=R_{\mathrm{s}}=70.0 \Omega$, all plots start close to the origin of the complex plane. The impedance spectrum at $280.0 \mathrm{mV}$ true potential (solid circles) consists of two approximate semicircles. According to the Nyquist stability criterion, at this true potential the system is 


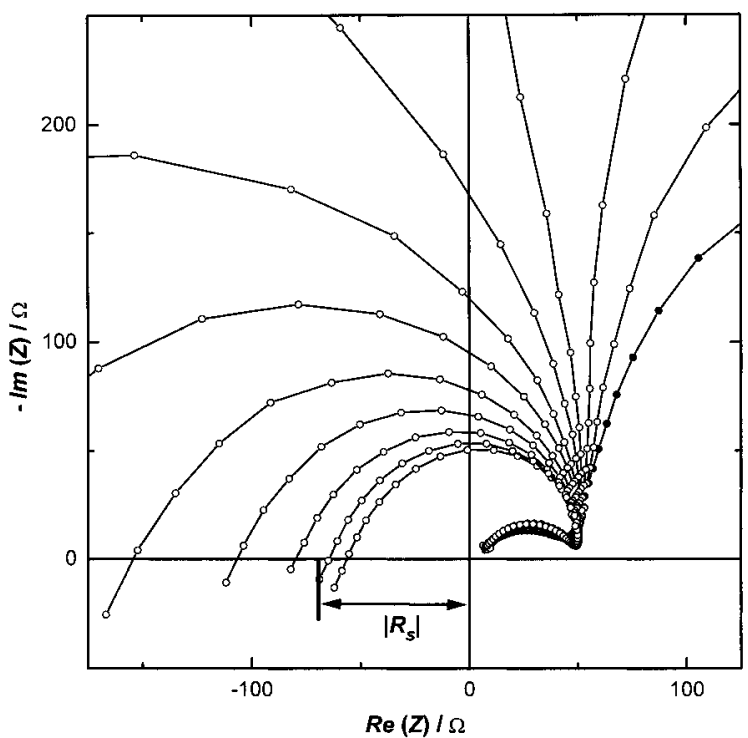

Figure 2. Impedance spectra of the $\mathrm{Cu}-\mathrm{SAGA}$ system at different electrode potentials. The first spectrum (solid circles) has been measured at $280.0 \mathrm{mV}$ by gradually decreasing the frequency from $17.0 \mathrm{kHz}$. Other spectra (open circles) have been determined at $280.0+n \times 10.0$ $\mathrm{mV}$ true anodic potentials $(n=1,2, \ldots, 11)$. Eight logarithmically equidistant points of a spectrum correspond to an order of magnitude decrease in the frequency ( 8 points/decade). Note that for the last two spectra the absolute values of the intersections with the real axis are smaller than $R_{\text {comp }}=R_{\mathrm{s}}=70.0 \Omega$.

unconditionally stable since the total impedance is always positive. Therefore, no current oscillations occur even if the $I R$ compensation is turned off. Note, however, that between 300 and $310 \mathrm{mV}$ the curvature of the second semicircle changes sign. As the potential is further increased, it becomes increasingly apparent that the impedance spectra would cross the negative part of the real axis at finite frequencies. Also, note that for the last two spectra the values of $|\operatorname{Re}(Z)|$ at the intersections are smaller than the compensated series resistance $R_{\mathrm{S}}=70.0 \Omega$.

Since turning off the $I R$ compensation results in only a horizontal shift of the impedance plot (in this case, $70 \Omega$ to the right), we conclude that a Hopf bifurcation would occur in the uncompensated system at a true potential value between 370 and $380 \mathrm{mV}$. Exactly at this critical potential (say, approximately $375 \mathrm{mV}$ ) the impedance spectrum of the uncompensated system would pass through the origin of the complex plane. As the frequency is finite at $Z_{\text {tot }}=0$, the conditions for a Hopf bifurcation are fulfilled, and the system is unstable. From the impedance spectra in Figure 2 we can also predict that at 380 and $390 \mathrm{mV}$ true potentials spontaneous current oscillations will occur in the uncompensated $\mathrm{Cu}-\mathrm{SAGA}$ system.

It is important to point out that the region of instability starts between 300 and $310 \mathrm{mV}$. But according to the impedance measurements, at true potentials less than approximately 375 $\mathrm{mV}$ the cell resistance $R_{\mathrm{s}}$ is not enough to destabilize the uncompensated system. To achieve the needed shift of the impedance plot (which would then result in current oscillations), a sufficiently large external resistor should be connected in series with the cell. In contrast to this conditional instability, the system is unconditionally stable at anodic potentials less than $300 \mathrm{mV}$, and it cannot be destabilized even by applying an external resistor.

At $440 \mathrm{mV}$ true electrode potential current oscillations are observed when the $I R$ compensation is turned off. When the compensation is then gradually increased, the system becomes
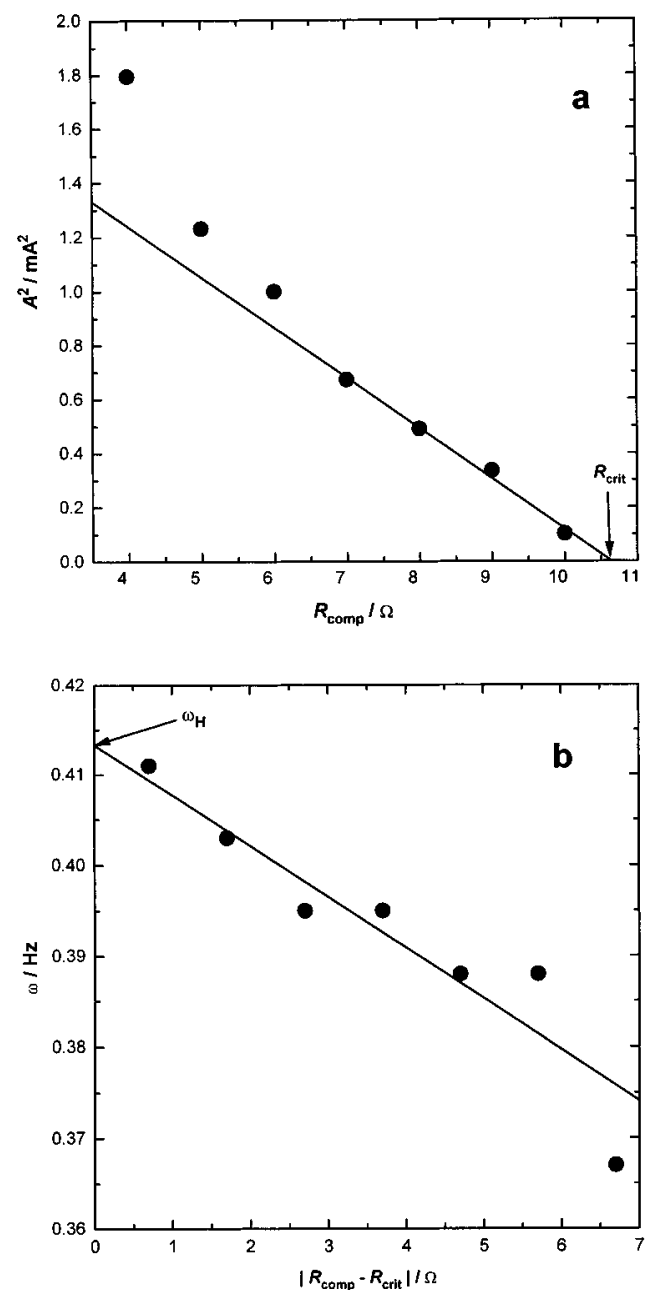

Figure 3. (a) Square of the amplitude of current oscillations in the $\mathrm{Cu}-\mathrm{SAGA}$ system as a function of the compensated resistance $R_{\text {comp }}$ $<R_{\mathrm{S}}=70.0 \Omega ; R_{\text {crit }}=10.7 \pm 0.8 \Omega$. (b) Frequency of oscillations as a function of $\left|R_{\text {comp }}-R_{\text {crit }}\right| ; \omega_{\mathrm{H}}=0.413 \pm 0.003 \mathrm{~Hz}$.

"less and less" unstable, and at some $R_{\text {crit }}<R_{\mathrm{S}}$ an inverse Hopf bifurcation takes place resulting in the loss of oscillations. As during Hopf bifurcations the amplitude scales with the square root of the bifurcation parameter, ${ }^{16}$ the critical value $R_{\text {crit }}=10.7$ $\pm 0.8 \Omega$ corresponding to the inverse Hopf bifurcation can be determined by extrapolating the fitted line shown in Figure $3 \mathrm{a}$ to zero amplitude. (Note that the linear range is too small, which makes the extrapolation quite difficult.) The frequency at the Hopf point is determined as shown in Figure $3 \mathrm{~b}$, giving $\omega_{\mathrm{H}}=$ $0.413 \pm 0.003 \mathrm{~Hz}$.

Alternatively, these critical values can be determined by simply measuring the frequency response of the fully compensated, stable cell at the given potential. The impedance spectrum of the $\mathrm{Cu}-\mathrm{SAGA}$ system at $440 \mathrm{mV}$ is shown in Figure 4a. The absolute value of the interpolated intersection with the real axis defines the minimal uncompensated series resistance corresponding to the Hopf bifurcation point: $R_{\mathrm{H}}=56.9 \Omega$. Since during impedance measurements the series resistance $R_{\mathrm{s}}$ $=70.0 \Omega$ has been fully compensated, it follows that an inverse Hopf bifurcation occurs at $R_{\text {crit }}=R_{\mathrm{s}}-R_{\mathrm{H}}=13.1 \Omega$. The finite frequency of oscillations at the bifurcation point, $\omega_{\mathrm{H}}=$ $0.422 \mathrm{~Hz}$, has been determined from the plot in Figure 4b. We conclude that the measurement of frequency responses gave us more accurate results in a considerably shorter time (and with much less effort), which clearly indicates the power of linear stability analysis by impedance spectroscopy. 

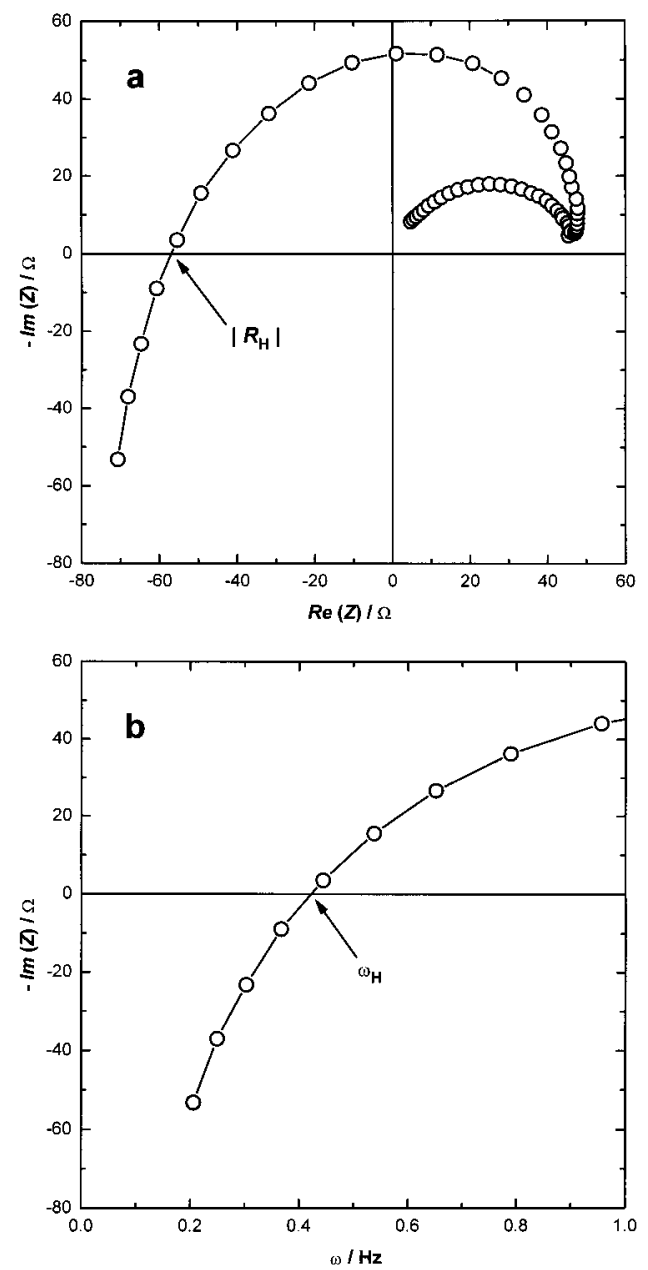

Figure 4. (a) Impedance spectrum (12 points/decade) of the $\mathrm{Cu}-$ SAGA system at $440.0 \mathrm{mV}$. The uncompensated series resistance at the Hopf point is $R_{\mathrm{H}}=56.9 \Omega$; (b) Imaginary part of the impedance $\operatorname{Im}(Z)$ as a function of the applied frequency; $\omega_{\mathrm{H}}=0.422 \mathrm{~Hz}$.

On the basis of impedance measurements at different true potentials, we have determined the position of the Hopf line in a diagram spanned by the minimal uncompensated series resistance $R_{\mathrm{H}}$ and the true electrode potential $E$. Results for the $\mathrm{Cu}-\mathrm{SAGA}$ system are shown in Figure 5a. (Note that for better visualization we have plotted $R_{\mathrm{H}}{ }^{-1}$.) It is important to stress that this figure is not a traditional bifurcation diagram as the horizontal axis represents a variable and not a bifurcation parameter. The true bifurcation parameter is the applied circuit potential including the ohmic drop. Note that inside the unstable region of the plot the true electrode potential is stable when full $I R$ compensation is applied, but it oscillates when the compensation is turned off. We adhere this unusual way of plotting the position of the Hopf line (by using constraints that can be well controlled) so that the experiments could be easily reproduced in other laboratories. The critical frequencies $\omega_{\mathrm{H}}$ by which oscillations would appear in the uncompensated system at different potential values are plotted in Figure 5b. The lower limit for oscillations is about $320 \mathrm{mV}$ true potential, in good agreement with Figure 2. It appears from Figure 5a that in this system the upper limit for the conditional instability is about $520 \mathrm{mV}$. However, as the true potential is increased to this "limit", the frequency of oscillations is approaching zero (Figure $5 b$ ), which prevents the accurate measurements within a considerable time frame.

The horizontal line in Figure 5a corresponds to the series resistance $R_{\mathrm{s}}$ of the applied electrochemical cell with the given
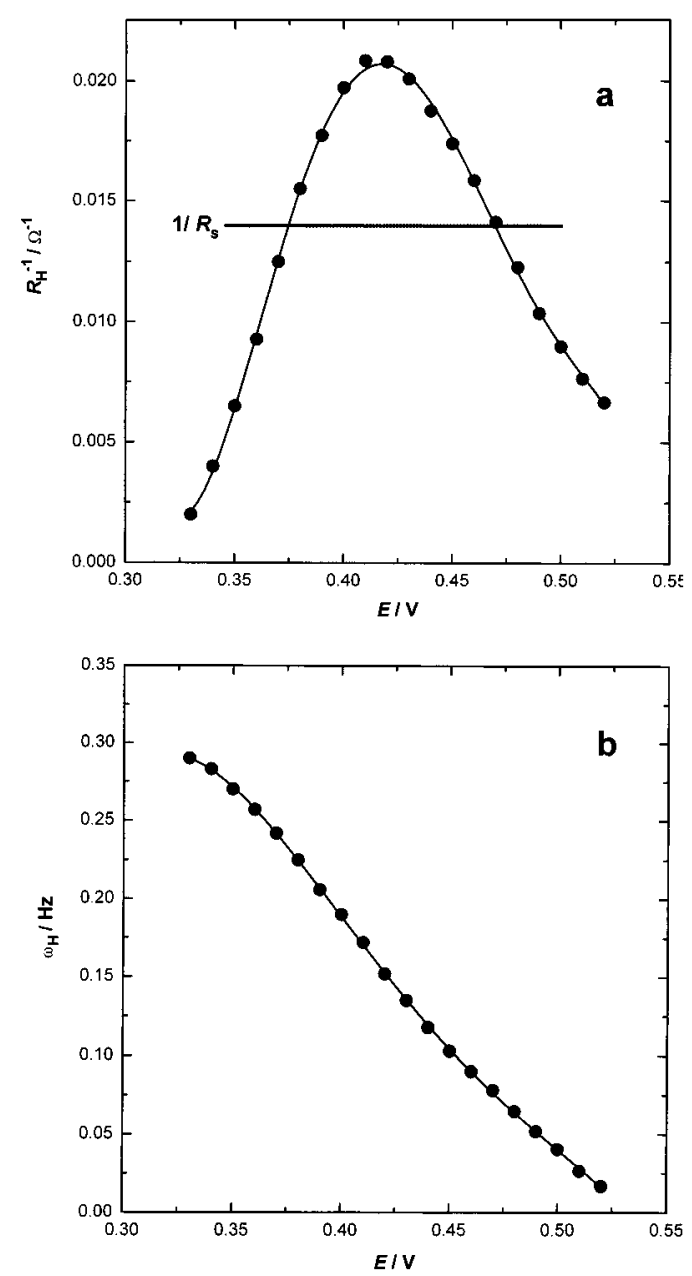

Figure 5. (a) Reciprocal values of $R_{\mathrm{H}}$ and (b) $\omega_{\mathrm{H}}$ values determined by the impedance analysis of the $\mathrm{Cu}-\mathrm{SAGA}$ system as a function of true electrode potential $E$. Horizontal line in (a) corresponds to the reciprocal series resistance $1 / R_{\mathrm{S}}$.

SAGA buffer. From its crossing with the Hopf line one can easily predict the potential range for unconditional (spontaneous) oscillations in the given $\mathrm{Cu}-\mathrm{SAGA}$ system under potentiostatic control. This system-without the addition of an external resistance-will show current oscillations between about 370 and $470 \mathrm{mV}$ true potentials. We note, however, that the position of the horizontal line is somewhat arbitrary, since the actual value of $R_{\mathrm{S}}$ depends on a number of technical details. In addition to the conductivity of the electrolyte, the series (ohmic) resistance $R_{\mathrm{S}}$ is also affected by the cell geometry, position of the electrodes, presence (or absence) of a separate compartment for holding the reference electrode, the construction of a glass frit or Luggin capillary connecting the compartments, etc.

It is important to point out that current oscillations are also possible outside the potential range defined by the horizontal line in Figure 5a. This requires only a lowered position of the horizontal line, which can be easily achieved by connecting a sufficiently large external resistance in series with the copper electrode. In principle, oscillations are possible at potential values larger than $520 \mathrm{mV}$, but the frequency of oscillations would be so small that, practically, no current oscillations could be observed. However, one should be very careful making predictions outside the range of measurements since other, for example, a saddle-node bifurcation, might also take place. At less than $310 \mathrm{mV}$ potential, however, the system cannot be destabilized even by applying an external resistance. At these 

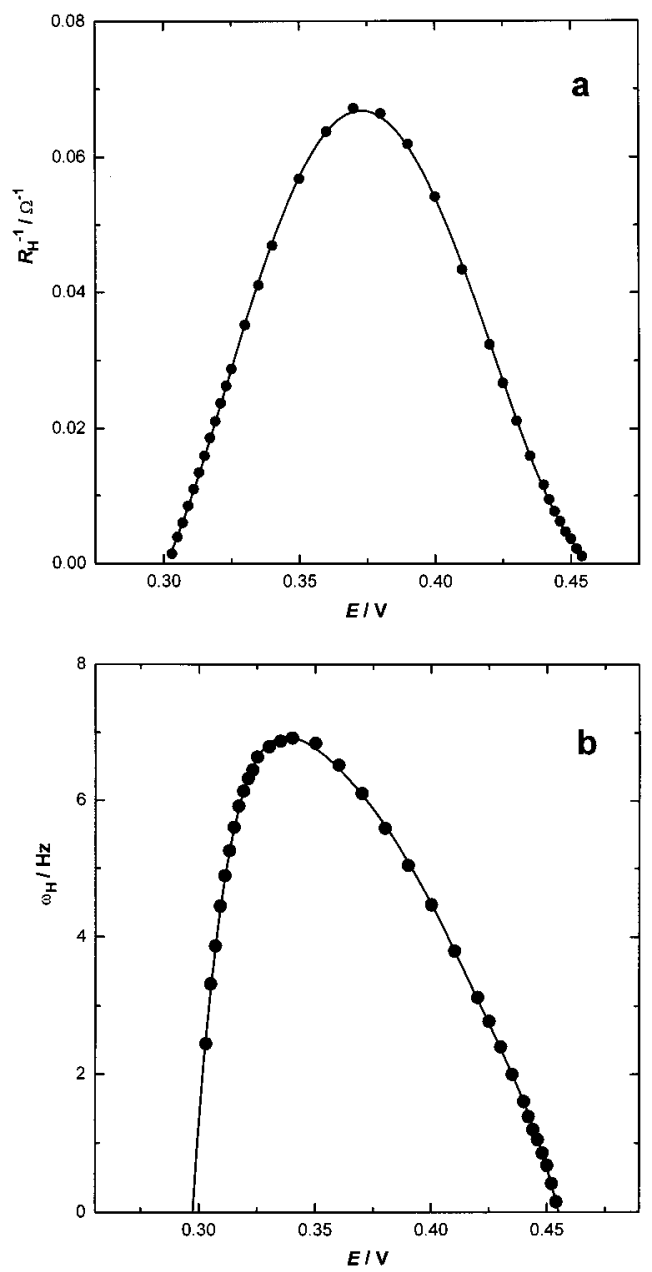

Figure 6. (a) Reciprocal values of $R_{\mathrm{H}}$ and (b) $\omega_{\mathrm{H}}$ values determined by the impedance analysis of the $\mathrm{Cu}-\mathrm{OP}$ system as a function of true electrode potential $E$.

potentials the system is unconditionally stable as that also follows from the impedance spectra shown in Figure 2.

Assuming that the mechanism of electrochemical processes in the $\mathrm{Cu}-\mathrm{SAGA}$ system does not change by varying the buffer composition, Figure 5a allows us to make predictions about the potential range for spontaneous current oscillations in different SAGA buffers. First, the new value of the series resistance $R_{\mathrm{S}}$ has to be determined by impedance spectroscopy; then the crossing points of the corresponding horizontal line with the Hopf line give the true potential range for current oscillations. Such predictions have been found in good agreement with our experimental findings.

The same type of impedance measurements were performed with the $\mathrm{Cu}-\mathrm{OP}$ system, too. Although the shape of $R_{\mathrm{H}}{ }^{-1}$ versus $E$ plot (Figure 6a) is similar to that found in the $\mathrm{Cu}-$ SAGA system, there is a considerable difference. Since the $R_{\mathrm{s}}$ value of this electrolyte is quite small $(7-12 \Omega$, only), the horizontal line $1 / R_{\mathrm{S}}$ (out of the plotted range) does not intersect with the Hopf line. Therefore, no spontaneous current oscillations are possible in the uncompensated $\mathrm{Cu}-\mathrm{OP}$ system. However, oscillations may occur if a sufficiently large external resistor is connected in series with the copper electrode. The experimentally found potential ranges for current oscillations have been found in good agreement with that predicted by Figure 6a. The full range of conditional instability determined by impedance spectroscopy is between 300 and $455 \mathrm{mV}$. Note that in the OP system the frequency apparently tends to zero at both ends of the instability interval (Figure 6b).

\section{Conclusions}

Impedance spectroscopy has been applied for the linear stability analysis of oscillatory electrodissolution of copper in acetate buffer and $o$-phosphoric acid electrolytes. Values of the minimum uncompensated series resistance $R_{\mathrm{H}}$ and the frequency $\omega_{\mathrm{H}}$ of oscillations at the bifurcation points have been determined by measuring the frequency responses of the fully compensated stable systems. The presence or absence of spontaneous current oscillations respectively in the acetate buffer and $o$-phosphoric acid electrolytes has been explained by the results of stability analysis based on impedance data. Although, from a traditional point of view, the two systems are different-one shows spontaneous oscillations while the other does not-we believe in that this is not essential. The main conclusion is that a sufficiently large series resistance is needed to destabilize the systems. Thus, the difference is arbitrary, and it is only of secondary importance whether the resistance is provided by the cell itself or it is externally connected to the circuit. For example, an external resistance would have been necessary to induce oscillations in both systems if a copper rod of sufficiently small diameter had been used.

Our observations clearly show the crucial role of series resistance $R_{\mathrm{S}}$ in the appearance of bifurcations leading to instabilities in a given electrochemical system. It is quite unfortunate that in a large body of research papers instability intervals have been reported without giving the $R_{\mathrm{S}}$ value of the particular system, which may prevent reproduction of the experiments in other laboratories. We stress that in future reports the accidental $R_{\mathrm{S}}$ values should always be given. The value of $R_{\mathrm{s}}$ and, perhaps, more importantly the minimal value of the uncompensated series resistance $R_{\mathrm{H}}$ corresponding to a given true potential can be easily determined by high-precision impedance analysis of the stable system.

The data shown in Figures 5 and 6 will be helpful in testing the suggested models for the complex chemical and transport processes taking place in the studied systems. It is clear, however, that such models could be successful only by incorporating the role of series resistance $R_{\mathrm{s}}$. In addition, the analysis of arcs and loops of the impedance spectra (not discussed in this paper) may also help in searching for the right models. Our future efforts will be concentrated along these lines by realizing that the difference in the impedance spectra of the studied systems must be closely linked to the difference in the chemical composition of surface layers ${ }^{6,17}$ at the electrode/ solution interface.

Finally, we note that with the help of impedance spectroscopy one may also locate regions in the parameter space where the system is unconditionally stable under potentiostatic control but can be made to oscillate under galvanostatic conditions. Preliminary experiments with the $\mathrm{Cu}-\mathrm{OP}$ system indicate that, indeed, the system can be made to oscillate at parameter values where the impedance data predict instabilities under galvanostatic control. This will be the subject of a future study.

Acknowledgment. This work was supported by the following Hungarian Grants: OTKA 17784, MKM 236, AKP 96145/20. The authors thank Marc T. M. Koper for helpful discussions. We are in debt to Tamás Pajkossy for valuable suggestions and critical reading of the manuscript.

\section{References and Notes}

(1) Fechner, G. Th. Schweigg J. Chem. Phys. 1828, 53, 129.

(2) Hudson, J. L.; Tsotsis, T. T. Chem. Eng. Sci. 1994, 49, 1493. 

411

(3) Shinbrot, T.; Grebogi, C.; Ott, E.; Yorke, J. A. Nature 1993, 363,

(4) Parmananda, P.; Sherard, P.; Rollins, R. W. Phys. Rev. E 1993, 47, R3003.

(5) Rollins, R. W.; Parmananda, P.; Sherard, P. Phys. Rev. E 1993, 47, R780.

6) Dewald, H. D.; Parmananda, P.; Rollins, R. W. J. Electrochem. Soc. 1993, 140, 1969

(7) Kiss, I. Z.; Gáspár, V.; Nyikos, L.; Parmananda, P. J. Phys. Chem. A 1997, 101, 8668

(8) Albahadily, F. N.; Ringland, J.; Schell, M. J. Chem. Phys. 1989, 90,813
(9) Schell, M.; Albahadily, F. N. J. Chem. Phys. 1989, 90, 822.

(10) Glarum, S. H.; Marshall, J. H. J. Electrochem. Soc. 1985, 132, 2872 2878.

(11) Sluyters-Rehbach, M. Pure Appl. Chem. 1994, 66, 1831

(12) Nyquist, H. Bell. Syst. Technol. J. 1932, 11, 126.

(13) Koper, M. T. M. Electrochim. Acta 1992, 37, 1771

(14) Koper, M. T. M. Adv. Chem. Phys. 1996, 92, 161.

(15) Koper, M. T. M. J. Electroanal. Chem. 1996, 409, 175.

(16) Andronov, A. A.; Vitt, A. A.; Khaikin, S. E. Theory of Oscillators; Pergamon: Oxford, 1966.

(17) Vidal, R.; West, A. C. J. Electrochem. Soc. 1995, 142, 2682, 2689. 